\title{
Critical slowing down in polynomial time algorithms
}

\author{
A. Alan Middleton \\ Department of Physics, Syracuse University, Syracuse, NY 13244
}

(October 31, 2018)

\begin{abstract}
Combinatorial optimization algorithms which compute exact ground state configurations in disordered magnets are seen to exhibit critical slowing down at zero temperature phase transitions. Using arguments based on the physical picture of the model, including vanishing stiffness on scales beyond the correlation length and the ground state degeneracy, the number of operations carried out by one such algorithm, the push-relabel algorithm for the random field Ising model, can be estimated. Some scaling can also be predicted for the $2 \mathrm{D}$ spin glass.
\end{abstract}

There has long been a close link between the concepts of statistical physics and the algorithms used to simulate condensed matter systems. The fundamental connection is the mathematics of graphs, which is applied analytically, for example, to compute high temperature series. Using sophisticated connectivity algorithms borrowed from computer science, one can numerically calculate quantities in percolation to a high precision [1]. The Fortuin-Kastelyn cluster representation [2] for magnets is the basis for the Swendsen-Wang algorithm [3]. By implementing nonlocal dynamics, these algorithms can greatly reduce the time needed for simulations near a phase transition. The study of disordered systems, such as spin glasses, pinned vortices in superconductors, and random field magnets, lead to the introduction of graphs with nonuniform edges [4,5]. Early in the study of disordered systems, it was realized that the study of such graphs is directly related to issues of computational complexity. In some cases [6], computing the ground state of a disordered material is computationally intractable, as the relevant optimization questions on a graph are NPhard [7]. Quite interestingly, some computationally intractable problems have been found to have phase transitions [8]. For example, given an ensemble of logical expressions characterized by the number of Boolean variables $N$ and clauses $M$, the fraction that are satisfiable can exhibit finite size scaling about a critical value of $M / N$.

In this letter, I present results on the behavior of ground state algorithms near phase transitions in two models, the random field Ising magnet (RFIM) and the 2D spin glass (2DSG), which, in contrast with problems such as satisfiability, are always solvable in time polynomial in the size of the graphs. These phase transitions lead to singularities in the mean time to solve the graph optimization problems. For the RFIM, a close connection is made between the critical slowing down of the ground state algorithm, the correlation lengths, and the degeneracy of the ground state in the thermodynamic limit. Numerical evidence is presented that suggests that the dynamic critical exponent is $z \approx 1$ for the RFIM; scal- ing arguments suggest $z \geq 1$. The behavior far from the transition can also be explained. The algorithm for the 2DSG is more difficult to analyze, but the observed uniform time per spin in the ferromagnetic (FM) phase is seen to be natural.

Generally, the dynamics at low temperature $T$ is exceedingly slow in disordered magnets, as seen in experiment and in Monte Carlo simulations using local moves [4]. The glassy dynamical behavior is attributed to the complex structure of the energy functional. The free energy barriers to equilibration at a length scale $\ell$ scale as $\ell^{\psi}$, so that the time to equilibrate a portion of the sample of size $\ell^{d}$ are expected to scale as $\sim \exp \left(\ell^{\psi} / T\right)$. However, for some random magnets there are algorithms which take time polynomial in $N$ to find an exact ground state. The process of finding the ground state uses "nonphysical" configurations or moves: a local search or simulated annealing that uses only physical configurations and local moves is hindered by the large energy barriers.

When using local Monte Carlo moves at finite $T$ to model uniform magnets, the relaxation or correlation time at continuous transitions scales as $L^{z}$, with $z_{\text {loc }} \geq$ $\gamma / \nu$ [9]. Nonlocal cluster moves, such as used in the Swendsen-Wang algorithm, can reduce the critical exponent $z$, with $z_{\mathrm{cl}} \geq \alpha / \nu$ [3], with $\alpha$ and $\nu$ the exponents for the heat capacity and correlation length, respectively. As the algorithms used to find the ground states of disordered magnets do not use local moves and are not designed to utilize clusters related to a critical point, it is less clear how many operations are required. Polynomial bounds on the worst case behavior of these algorithms do exist. For graphs with $n$ vertices and $m$ edges, the highest level version of the push-relabel (PR) algorithm, used here for the RFIM, will use $O\left(n^{2} \sqrt{m}\right)$ time. Useful algorithms for general matching [10], applied here to the 2DSG, use from $O\left(n^{3 / 2}\right)$ to $O\left(n^{3}\right)$ operations, up to logarithmic corrections, assuming $m \propto n$. In practice, however, for typical disorder realizations of physical interest in finite dimension $d$, these algorithms are much faster, with the average running time for many problems scaling roughly as $N^{q}$, with $q$ typically in the range $1.1-1.3$ 
[5,11. For systems with a single $T=0$ fixed point, such as the elastic medium in a random potential [11], this scaling for the time would not be expected to vary with parameters.

The $T=0$ RFIM has a phase transition that has been extensively studied [12 using a mapping 113 of the ground state to the optimization problem max-flow [14]. The Gaussian RFIM has Hamiltonian $H_{R}=$ $-J \sum_{<i j>} s_{i} s_{j}-\sum_{i} h_{i} s_{i}$, where the allowed spin values on $N=L^{d}$ lattice sites $i$ are $s_{i}= \pm 1$, the ferromagnetic couplings $J$ are positive, and the random fields $h_{i}$ are Gaussian distributed, with mean 0 and variance $\Delta^{2} J^{2}$. In the paramagnetic phase $\left(\Delta>\Delta_{c} \approx 2.27\right)$, the spins are pinned by the external fields $h_{i}$ and the magnitude of the net magnetization $m=N^{-1} \sum s_{i}$ vanishes as $L \rightarrow 0$. In the ferromagnetic phase $\left(\Delta<\Delta_{c}\right)$, the ferromagnetic coupling $J$ dominates and $|m|$ has a finite limit, with the sign of $m$ determined roughly by the total of $\sum h_{i}$ (some spins will be reversed by fluctuations in $h_{i}$.) Results for the time for this algorithm to find the ground state in a $3 \mathrm{D}$ cubic lattice with periodic boundaries are plotted as a function of $\Delta$ and linear system size $L$ in Fig. 1. Plots of the number of primitive operations executed are nearly identical in form. Near the phase transition separating the ferromagnetic from paramagnetic phase, the time to find the ground state shows a characteristic critical slowing down. This notable effect reflects the deep connection between the dynamics of the ground-state algorithm and the physics of the RFIM.

The application of max-flow algorithms to the RFIM is well established, but to make the connection between scaling in the RFIM and algorithm timing, it is useful to review the algorithm. The network flow algorithm generally used to solve the RFIM, because of its speed, is the PR algorithm of Tarjan and Goldberg [15]. The algorithm described here uses a modification that removes the need for source and sink nodes [16], reducing memory usage and also clarifying the physical connection.

The modified PR algorithm starts by assigning an "excess" $x_{i}$ to each lattice site $i$, with $x_{i}=h_{i}$. Residual capacity variables $r_{i j}$ between neighboring sites are initially set to $J$. A height variable $u_{i}$ is then assigned to each node via a global update step. In this global update, the value of $u_{i}$ at each site in the set $\mathcal{T}=\left\{j \mid x_{j}<0\right\}$ of negative excess sites is set to zero. Sites with $x_{i} \geq 0$ have $u_{i}$ set to the length of the shortest path, via edges with positive capacity, from $i$ to $\mathcal{T}$.

The ground state is found by successively rearranging the excesses $x_{i}$, via "push" operations, and updating the heights, via "relabel" operations. When no more pushes or relabels are possible, a final global update determines the ground state: those sites which are path connected by bonds with $r_{i j}>0$ to $\mathcal{T}$ have $s_{i}=-1$, while the sites which are disconnected from $\mathcal{T}$ have $s_{i}=1$. A push operation moves excess from a site $i$ to a lower height neighbor $j$, if possible, that is, whenever $x_{i}>0, r_{i j}>0$ and $u_{j}=u_{i}-1$. In a push, the working variables are modified according to $x_{i} \rightarrow x_{i}-\delta, x_{j} \rightarrow x_{j}+\delta, r_{i j} \rightarrow r_{i j}-\delta$, and $r_{j i} \rightarrow r_{j i}+\delta$, with $\delta=\min \left(x_{i}, r_{i j}\right)$. Push operations tend to move the positive excess towards sites in $\mathcal{T}$. When $x_{i}>0$ and no push is possible, the site is relabeled, with $u_{i}$ increased to $1+\max _{\left\{j \mid r_{i j}>0\right\}} u_{j}$. In addition, if a set of highest sites $\mathcal{U}$ become isolated, with $u_{i}>u_{j}+1$, for all $i \in \mathcal{U}$ and all $j \notin \mathcal{U}$, the height $u_{i}$ for all $i \in \mathcal{U}$ is increased to its maximum value, $N$, as these sites will always be isolated from the negative excess nodes. A proof of the correctness of the PR flow algorithm can be found in standard textbooks 14 and its application to the RFIM is well known [5]. Periodic global updates, here applied every $N$ relabels, are often crucial to the practical speed of the algorithm. The highest site heuristic is used here, which applies pushes and relabels where $u_{i}$ is maximal and $x_{i}>0$.

The PR algorithm is intuitively appealing: when the initial capacities within a region are large, the excess can be rearranged so that the positive excesses cancel the negative excesses as much as possible. The remaining excess values having the sign of the original total excess for the region. As $r_{i j}=J$ and $x_{i}=h_{i}$ initially, large capacities correspond to the ferromagnetic bonds being strong enough to favor alignments of the spins, with the spin direction given by the sign of the total $h_{i}$ for the region. If the initial capacities are not large enough (weaker $J$ ), the regions align independently, according to the local field, and the excesses do not cancel. The number of steps needed to move excess across the diameter of a region via push operations is bounded below by the linear size of the region. Note that the running time in the FM phase is somewhat dependent on the majority magnetization, due to the up/down asymmetry of the algorithm.

I now argue that these correspondences can be used to bound the running time of the algorithm, using the physical properties of the RFIM, particularly the behavior of the ground state degeneracy in the thermodynamic limit. Recent work [12] has given strong numerical evidence of insensitivity of the interior spins in the ground state to boundary conditions, when $\Delta>\Delta_{c}$. (This is in contrast with the scenario for, e.g., mean field [4] or highly disordered 17] spin glasses in $d>8$, where the entire solution is sensitive to the volume.) Most importantly, this implies that the ground state solution is determined by the $h_{i}$ within a volume typically of the size of the correlation volume $\xi^{d}$. However, in the FM phase, the interior is sensitive to the boundaries and a finite fraction of the spins flip infinitely often as the sample volume is increased, as the net magnetization is given by a coarse-grained global sum of the $h_{i}$.

First, consider the case $\Delta \gg 2 d$, where, in the ground state, almost all spins satisfy $s_{i}=\operatorname{sgn}\left(h_{i}\right)$. In the algorithm, the initial positive excesses at sites $i$ where $h_{i}>2 d J$ remain positive, as the total capacity of bonds 
leaving the site is only $2 d J$, so that push operations can only reduce $h_{i}$ by $2 d J$. The sites with excess $x_{i}<-2 d J$ also always have negative excess. There will be some small rearrangement, but only locally, and the sign of the excess will change only at a very few sites during the execution of the algorithm, which terminates after a number of operations $\propto N$ (up to logarithmic corrections 18.)

A similar scenario holds, but at scales $\xi$, for $\Delta>\Delta_{c}$. The algorithm establishes the boundaries of correlation volumes by rearrangement of excess over distances of scale $\xi$. Further rearrangement is blocked by the effective decrease in residual capacity with scale (as the stiffness, corresponding to the scale dependent $J$, decreases rapidly on scales greater than $\xi[19]$.) The question to be answered, then, is how long the algorithm takes, per spin, to redistribute excess on scale $\xi$. The number of push and relabel operations in a volume $\xi^{d}$ is bounded below by $\xi^{d+1}$. For the excess to be pushed over a distance $\xi$, the relative heights must differ by at least $\xi$, so that at least $O\left(\xi^{d+1}\right)$ relabels $R$ must be performed for each correlation volume (global updates lead to height changes, but these do not appear to affect the scaling, empirically.) This gives the estimate $R \sim L^{3} \xi$. This scaling is consistent with numerical results, up to logarithmic corrections, for $d=1,3$. The inset to Fig. 1 1 show a scaling plot for $R$, for example, with $\left(R L^{-3}\right)^{1 / \nu} \delta$ plotted as a function of the finite size scaling variable $\delta L^{1 / \nu}$, $\delta=\left(\Delta-\Delta_{c}\right) / \Delta_{c}$, with the values $\nu=1.37$ and $\Delta_{c}=2.27$ fixed parameters, determined independently [12]. A fit in $d=1$, with $\nu=2$ and $\Delta_{c}=0$ also fixed by known results, describes the data well for $L \leq 5 \times 10^{6}$, with $R \sim L \xi \ln (L / \xi)$ (without the global relabeling heuristic.)

One limit where the asymptotic time for the algorithm can be described more precisely is when $\Delta \ll$ $[L \ln (L)]^{-1 / 2}$.) Here, the capacities do not limit the rearrangement of excess: the final state is either $x_{i} \geq 0$ everywhere or $x_{i} \leq 0$ everywhere, corresponding to a uniform $s_{i}= \pm 1$ state, according to whether $\sum h_{i} \geq 0$ or $\sum h_{i} \leq 0$, respectively. The dynamics of the PR algorithm is set by the fluctuations in the total $h_{i}$ in regions at each length scale. At any time scale of the computation, the positive excess will be pushed towards the nearest negative excess region, with the distribution of excess negative or positive over a particular volume with $\ell^{d}$ sites, as the rearrangement of excess will have been completed over shorter lengths at an earlier time scale. Generally, but especially given sufficiently frequent global updates, the sites with greatest $u_{i}$ will be furthest from the set $\mathcal{T}$. As the highest sites are examined for pushing, the excess will be moved from these sites to the next lower level. These sites will then have their excess moved to the next lower level and the algorithm will "sweep" the excess through the volume of diameter $\approx 2 \ell$. This will establish a distribution of excess that will have uniform sign over a region of size $2 \ell$. In this fashion, the algorithm will solve for the sum of $h_{i}$ recursively. The number of steps at each stage will be $L^{d}$ and $O(\ln L)$ stages will be required, giving a total running time $\sim L^{d} \ln (L)$. This result is consistent with the numerical timings for very small $\Delta$, with the data for $R L^{-d}$ linear in $\ln (L)$ over sample dimensions $16 \leq L \leq 128$ to within the $1 \%$ numerical error for $d=3$ and over $10^{3} \leq L \leq 5 \times 10^{6}$ to within the same error for $d=1$. Coarsening of the height variables during the algorithm is displayed in Fig. 2.

To indicate the generality of critical slowing down and partial arguments for other systems, it is useful to compare the RFIM results with results for the 2DSG. The Hamiltonian is $H_{S}=\sum_{<i j>} J_{i j} s_{i} s_{j}$, where again the $s_{i}$ are Ising spins. The Gaussian distributed $J_{i j}$ have mean $J_{0}$ and variance 1 . The SG to FM transition 20] takes place as $J_{0}$ is increased through the critical value $J_{c} \approx 0.96$. The mapping from the 2DSG with free boundaries to a general matching problem is given in Ref. [6]: energy is minimized by selecting a state with a minimum total weight for frustrated bonds. Timing results for the 2DSG are shown in Fig. 3. One notable difference from the RFIM is the apparent convergence to constant time per spin in the FM phase. As the algorithm used for the 2DSG uses a bond representation, the algorithm does not need to distinguish degenerate states related by global spin flips. If the frustration is low enough, the FM phase is obtained by local operations giving a solution with percolating unfrustrated bonds. For low $J_{0}$, in the SG phase, in contrast, though locally the ground state is insensitive to the boundaries, the global ground state is sensitive to the disorder and the operations (augmenting paths) must therefore be carried out over all scales, so that the time per spin may diverge as a power of $L$ (numerically, approximately as $L^{0.78 \pm 0.10}$ for $96 \leq L \leq 720$ and $J_{0}=0$.) At the critical point, independent calculations 18] show that the domain wall fractal dimension increases slightly, so that bond updates on large scales are more expensive.

In summary, the time for a polynomial time algorithm to find ground states is examined near zero temperature critical points for two models, the random field Ising model and the 2D spin glass. At the critical points, the combinatorial optimization algorithms used, PR flow and general matching, while exponentially faster than, say, simulated annealing at finding the ground state, slows down. This slowing down of the algorithm is argued to be closely related to important physical ideas, namely, the uniqueness or two-fold degeneracy of the ground state in various phases and the divergence of the correlation length as the transition is approached. The time for the algorithm can be understood in detail for the RFIM with small random fields. It will certainly be of interest to consider such slowing down and scaling at other fixed points for other polynomial time algorithms, to expand our understanding of the physics and algorithms for these systems. These considerations are clearly relevant 
for developing efficient parallel algorithms. I would like to thank J. Machta for useful discussions. This work was supported in part by the NSF (DMR-9702242.)

[1] See, e.g., M. E. J. Newman and R. Ziff, condmat/0101295.

[2] C. M. Fortuin and P. W. Kastelyn, Physica 57, 536 (1972).

[3] R. H. Swendsen and J.-S. Wang, Phys. Rev. Lett. 58, 86 (1987); X.-J. Li and A. D. Sokal, Phys. Rev. Lett. 63, 827 (1989).

[4] Spin Glasses and Random Fields, A. P. Young, ed., (World Scientific,Singapore, 1998); K. Binder and A. P. Young, Rev. Mod. Phys. 58, 801 (1986).

[5] M. J. Alava, et al, in Phase Transitions and Critical Phenomena, Vol. 18, C. Domb and J. L. Lebowitz, eds (Academic Press, San Diego, 2001).

[6] For the case of the spin glass, see F. Barahona, J. Phys. A 15, 3241 (1982).

[7] C. H. Papadimitriou, Computational Complexity, (Addison-Wesley, 1994).

[8] For the distinct behaviors of heuristics with typical polynomial and exponential times, see R. Monasson, et al, Nature 400, 133 (1999).

[9] K. Kawasaki, in Phase Transitions and Critical Phenomena, C. Domb and M.S. Green, eds. (Academic, New York, 1972), Vol. 2.

[10] W. Cook and A. Rohe, INFORMS J. Comp. 11, 138 (1999).

[11] A. A. Middleton, Phys. Rev. E 59, 2571 (1999);

[12] A. T. Ogielski, Phys. Rev. Lett. 57, 1251 (1986); A. K. Hartmann and U. Nowak, Eur. Phys. J. B 7, 105 (1999); D. S. Fisher and A. A. Middleton, in preparation.

[13] J. C. Angle d'Auriac, M. Preissmann and R. Rammal, J. Phys. Lett. 46, L173, (1985).

[14] T. H. Cormen, C. E. Leiserson and R. L. Rivest, Introduction To Algorithms, (MIT Press, Cambridge, MA, 1990).

[15] A. V. Goldberg and R. E. Tarjan, J. Assoc. Comput. Mach. 35, 921 (1988); B. V. Cherkassky and A. V. Goldberg, Algorithmica 19, 390 (1997).

[16] The use of negative excesses is also useful in min-cost flow algorithms.

[17] C. M. Newman and D. S. Stein, Phys. Rev. Lett. 72, 2286 (1994).

[18] A. A. Middleton, unpublished.

[19] A feature of the ground state for dimensions $d>2$ is that both positive and negative spin domains can percolate for $h>h_{c}$ (see J. Esser, U. Nowak, and K. D. Usadel, Phys. Rev. B 55, 5866 (1997) and Ref. 12].) This does not indicate a long-range stiffness: the correlation volumes are isolated. Boundary changes or local changes in disorder penetrate only a distance $\xi$ in the paramagnetic state.

[20] W. L. McMillan, Phys. Rev. B 29, 4026 (1984).

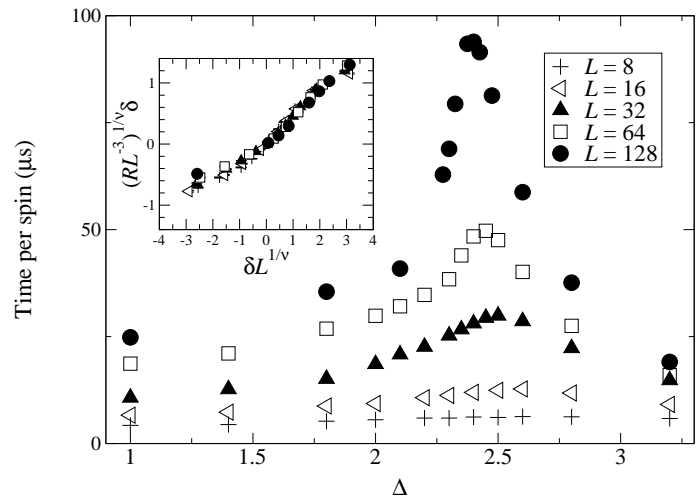

FIG. 1. The CPU time needed to find the ground state in the 3D RFIM, for a $766 \mathrm{MHz}$ PIII. The inset plots $\left(R L^{-3}\right)^{1 / \nu} \delta$, the scaled number of relabel operations per site, with $\delta=\left(\Delta-\Delta_{c}\right) / \Delta_{c}$, vs. the finite-size scaling variable $\delta L^{1 / \nu}$. The values $\Delta_{c}=2.27$ and $\nu=1.37$ are not fit parameters, but are derived independently. Statistical error bars are about $1 / 5$ of the symbol size in both plots.
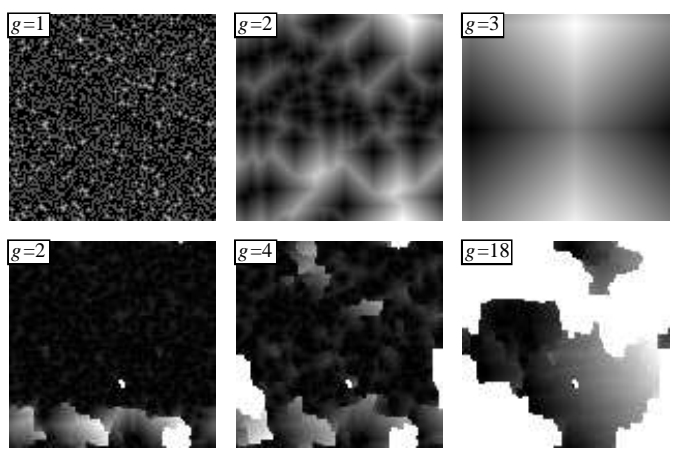

FIG. 2. Images of the heights $u_{i}$ at intermediate stages of the PR algorithm, in the 2D RFIM for $100^{2}$ samples, for $\Delta=10^{-5}$ (top) and $\Delta=1.0$ (bottom). In the latter case, coarsening is cutoff by the finite correlation length; the visible spin-up domain shows some structure. The shading is darkest at the maximum $u_{i}$ and is white where $u_{i}=0$. The number of global updates, $g$, is given by the labels.

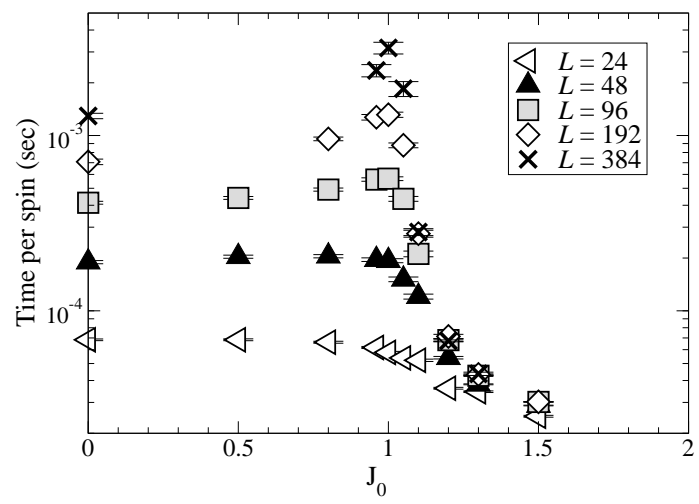

FIG. 3. The CPU time needed to find the ground state in the $2 \mathrm{D}$ spin glass, as a function of the ferromagnetic strength $J_{0}$ and system size $L$. 